\title{
Gastric cancer with liver metastasis
}

\author{
Marek V ${ }^{1}$, Zahorec $\mathrm{R}^{2}$, Palaj $\mathrm{J}^{1}$, Durdik $\mathrm{S}^{1}$ \\ Department of Surgical Oncology, St. Elizabeth Hospital, Medical School of Comenius University, Bratislava, \\ Slovakia. vitezslav.marek@gmail.com
}

\begin{abstract}
Surgical treatment of gastric cancer with liver metastasis (GCLM) is currently a frequent topic of discussion at professional surgical symposia. There is a low number of patients and a lack of large clinical multi-center studies describing the benefits of this treatment approach.

The article describes a patient with GCLM, growing through stomach wall serosa, invading the spleen hilum, distal part of pancreas with metastasis to $\mathrm{S} 7$ of the right liver lobe. The patient had total gastrectomy performed with D2 lymphadenectomy, distal pancreatectomy with splenectomy, resection of diaphragm and RFA of the metastatic lesion in S7 of the liver. Post-surgery course was free of complications, followed by adjuvant chemotherapy. 2 years after the surgery, the patient is in full remission, free of any relapse.

Liver resection or RFA is not commonly used in the gastric cancer with liver metastasis (GCLM). At present, there is no direct marker available to define the degree of biological aggressiveness of the tumor (indicating or contra-indicating the surgical treatment), therefore we are left to rely on indirect prognostic factors: cancer invasion in the gastric wall serosa, presence of 3 and more liver metastases, size of metastasis exceeding $50 \mathrm{~mm}$ (Fig. 2, Ref. 13). Text in PDF www.elis.sk.

KEY WORDS: GCLM-gastric cancer liver metastasis, RFA-radiofrequency ablation.
\end{abstract}

\section{Introduction}

Gastric cancer is the fourth most common cancer worldwide (1). Early diagnosis of earlier stages of the disease with adequate treatment / R0 resection of stomach + D2 lymphadenectomy + suitable perioperative chemotherapy/ brings a better outlook (2).

Remote metastases as a sign of systemic disease reduce the overall patient survival. The most common site for gastric cancer metastasis is the liver (3).

For the sake of comparison, the liver resection is currently accepted as a treatment for liver metastases of colorectal cancer with referred 5-year survival in $40-56 \%$ of patients (4). Thanks to advances in surgical techniques and perioperative chemotherapy, the indication range keeps expanding.

Compared to colorectal cancer, the gastric cancer represents a more aggressive cancer disease with heterogenic nature (5).

Other metastatic lesions associated with gastric cancer such as: peritoneal carcinomatosis or extensive involvement of the regional lymph nodes, significantly deteriorates the patient's outcome, contraindicating the surgical treatment.

GCLM is considered a systemic disease with adverse outcome and systemic chemotherapy is indicated as the first line of treatment (6)

${ }^{1}$ Department of Surgical Oncology, St. Elizabeth Hospital, Medical School of Comenius University, Bratislava, Slovakia, and ${ }^{2}$ Department of Anesthesiology and Intensive medicine, St. Elizabeth Hospital, Medical School of Comenius University, Bratislava, Slovakia

Address for correspondence: V. Marek, MD, Department of Surgical Oncology, St. Elizabeth Hospital, Heydukova 10, SK-812 50 Bratislava, Slovakia.
Thanks to the effort on the part of some of the surgeons to reverse the adverse outcome in resectable GCLM, who performed resection or RFA surgery on the liver, we were able to collect interesting outcomes -5 -year survival of $0-45 \%$ of patients $(7,8)$.

These studies are greatly handicapped by the low number of patients, mostly from a single center (7).

Our case report presents a GCLM patient with systemic disease and very poor outlook. Nevertheless, he had surgery performed and his postoperative course was a very pleasant surprise for him and for us as well.

\section{Clinical report}

59-year old patient was admitted to the clinic of cancer surgery of the St. Elizabeth Cancer Hospital Bratislava on 14.2.2013, with adenocarcinoma of the proximal third of the stomach verified by gastrofibroscopy and histology.

CT of chest and abdomen (10.2.2013) identified a large exulcerated tumor of the proximal third of the stomach with diameter of $100 \mathrm{~mm}$, growing into the hilum of the spleen. The tumor was in wide contact with the left diaphragm, with suspect infiltration. The tumor was not spreading to esophagus, was in contact with distal pancreas and left suprarenal gland. In the right liver lobe S7, there was a metastatic lesion $52 \mathrm{~mm}$ in diameter, caudal displacement of the left kidney into the pelvis, there were no signs of ascites or retroperitoneal lymphadenopathy.

The laboratory image included the elevation of tumor markers: CEA: 226 ng/l, Ca 19-9:2439 U/ml, Ca 72-4: 440 U/ml. Other laboratory parameters were normal. 


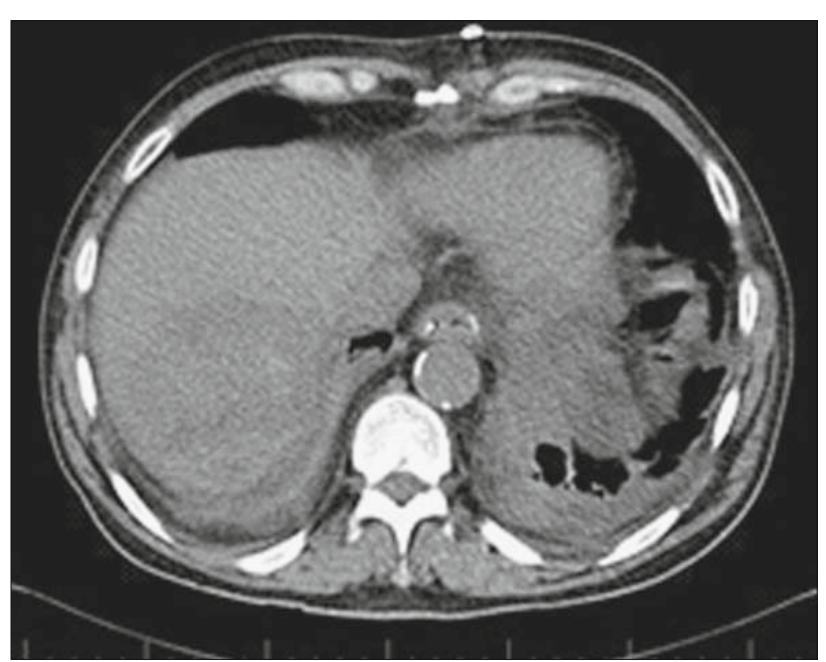

Fig. 1. Postoperative CT of abdomen - found a destroyed metastatic lesion in liver lobe S7.

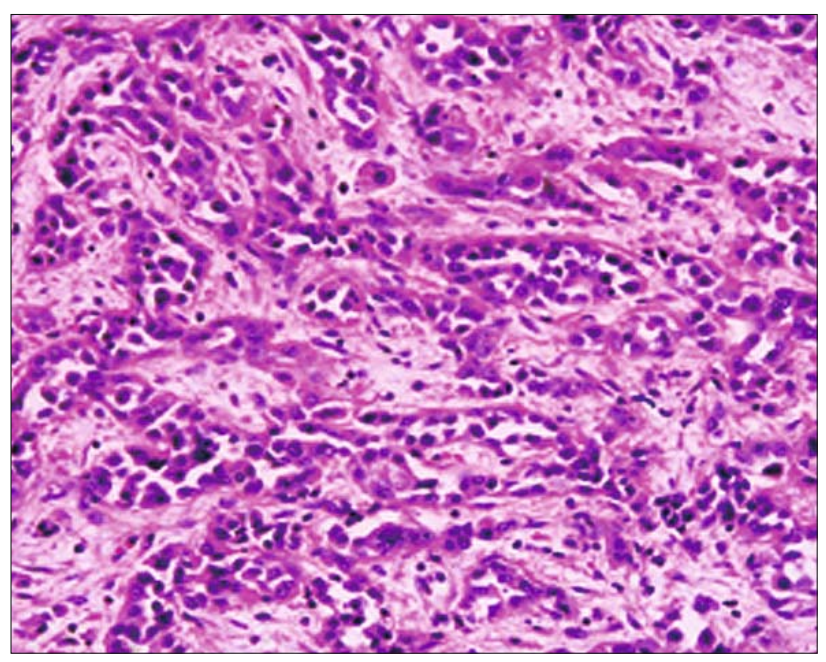

Fig. 2. Mucinous adenocarcinoma of the subcardial part of the stomach with lower differentiation. KRAS status: wildtype, BRAF status: wild-type.

The patient was indicated for a surgical intervention. On February 15, 2013 the patient was operated on. During inspection of the abdominal cavity, a large tumor of the superior third of the stomach was found, growing into the spleen hilum, distal pancreas, left suprarenal gland and left part of the diaphragm. In the right liver lobe, in 7th segment, there was a metastatic lesion, 52 $\mathrm{mm}$ in size. There was no peritoneal carcinomatosis or ascites, no lymphadenopathy in the truncus coeliacus (celiac artery) drainage area or in retroperitoneal area.

Multivisceral resection was performed in the following extent - total gastrectomy with D2 lymphadenectomy, distal pancreatectomy with splenectomy, left-side adrenalectomy, resection of diaphragm. Due to the extent of the multivisceral resection resulting in significant surgical burden on the organism, the hepatectomy was not indicated, the metastatic lesion was destroyed using RFA in 2x12 minutes mode. In the second stage, liver resection is planned
- right-sided hepatectomy.

There were no complications in the post-operative period. The follow-up post-operative CT of abdomen on 21-2-2013 - found a destroyed metastatic lesion in liver lobe S7, without traces of viable tumor - the planned re-operation with secondary liver resection was cancelled (Fig. 1).

Histology - mucinous adenocarcinoma of the subcardial part of the stomach with lower differentiation, deep penetration in spleen hilum (about $20 \mathrm{~mm}$ ) - pT4bN1M1 (Fig. 2).

Gastric mucosa surrounding the tumor had signs of chronic gastritis. A total of 20 lymph nodes have been examined, two were found to contain metastasis of the adenocarcinoma.

The tissue of pancreas and left suprarenal gland was free of tumor changes

Adjuvant chemotherapy was initiated from March to August 2013 - a combination of cis platinum + 5-fluorouracil (6 cycles). The patient is surviving 2nd year after surgery, without signs of disease recurrence, in a complete remission with normal tumor marker levels.

\section{Discussion}

Liver resection / RFA is not a frequent treatment modality for gastric cancer with liver metastasis (GCLM). This is well documented by a Korean study, where in $10 \%$ of the 100,000 GCLM patients, only $4 \%$ had surgery. At present, there is no clear consensus supporting liver resection in this type of tumor.

In this respect the study of Kinoshita et.al has become a breakthrough (10). It describes a 5-year disease-free survival in $30 \%$ of carefully selected patients. This confirmed, that a small subpopulation of patients with GCLM may benefit from liver resection or RFA. The median recurrence-free survival time was 9 months.

Half of the patients had recurrence within 1 year, in spite of $\mathrm{R} 0$ resection and careful selection. On the other hand, there was sufficient number of patients with long-term survival. This can be explained by varying tumor sub-populations with differing biological behavior (10).

The question is: Which GCLM patients are suitable for surgical intervention?

At present, there is no direct marker available, defining the degree of biological aggressiveness of the tumor (indicating or contra-indicating the surgical treatment), therefore we are left to rely on indirect prognostic factors - number of liver metastases, size of metastatic lesion (7).

Several studies have attempted to identify the prognostic factors defining an adverse outlook for patients and contraindicating surgical intervention.

Among these studies, the multi-center study by Japanese authors stands out (10). This study defines 3 adverse prognostic factors:

1) invasion of serosa by primary tumor,

2) 3 and more liver metastases,

3) size of liver metastasis exceeding $50 \mathrm{~mm}$.

The study noted a significant difference in survival between patients without a prognostic factor and patients with one of the 
three prognostic factors. The authors recommended to consider surgical intervention in the presence of any of the three risk factors.

Patients with a lower number of risk factors had better 3 and 5-year survival following liver resection (10).

The indication for surgical intervention in the GCLM is subject to overall clinical condition of the patient, but liver resection should definitely be contraindicated in the presence of all three adverse prognostic factors (no long-term survival was noted) (10).

Micrometastases, which were not identifiable during primary intervention, are considered to be the most common cause of the recurrence of liver metastasis in the GCLM.

Repeated hepatectomy was performed only in $14.4 \%$ of patients, which is significantly lower number of hepatectomies compared to the patients with colorectal cancer. This is caused by a different pathophysiological course of gastric cancer relapse.

Hepatic resection is presently considered to be justified only in a case of solitary relapsing metastasis of the GCLM (11).

The role of chemotherapy in the GCLM is not clearly defined. Neo-adjuvant chemotherapy is being brought forward, that can be used to differentiate responders from non-responders. Surgical intervention is contraindicated in non-responders.

GCLM patients treated by systemic chemotherapy alone have $1.7 \%$ 5-year survival (12).

Several studies assessed the use of RFA in GCLM, recommending it for solitary lesions up to $30 \mathrm{~mm}$ in size, located in the periphery of the liver. No clear advantage of RFA compared to surgical resection has been shown (13).

\section{Conclusion}

The number of patients with clinically resectable GCLM is low. A careful selection of patients with R0 resection ensures longterm survival in a complete disease remission.

\section{References}

1. International Agency for Research on Cancer . GLOBOCAN 2012: Estimated cancer Incidence, Mortality and Prevalence Worldwide in 2012. http://globocan.iarc.fr/Pages/fact-sheets-population.aspx.

2. Tanaka M, Ma E, Tanaka H, Ioka A, Nakahara T, Takahashi H. Trends of stomach mortality in Eastern Asia in 1950-2004: comprehensive study of Japan, Hong Kong and Singapore using age, period and cohort analysis. Int J Cancer 2012; 130: 930-936.

3. Sakamoto Y, Ohyama S, Yamamoto J, Yamada K, Seki M, Ohta K et al. Surgical resection of liver metastases of gastric cancer: analysis of a 17-year experience with 22 patients. Surgery 2003; 133: 507-511.

4. Jong MC, Pulitano C, Ribero D, Sturb J, Mentha G, Schulick RD et al. Rates and patterns of recurrence following curative intent surgery for colorectal liver metastasis an international multi-institutional analysis of 1669 patients. Ann Surg 2009; 250: 440-448.

5. Kodera Y, Fujinati K, Fujinati N, Ito S, Muro K, Ohashi N et al. Surgical resection of hepatic metastasis from gastric cancer: a review and new recommendation in the Japanese gastric cancer treatment guidelines. Gastric Cancer 2014; 17: 206-212.
6. Japanese Gastric Cancer Association. Japanese gastric cancer treatment guidelines (Ver.3).gastric Cancer 2011; 14: 113-123.

7. Takemura N, Saiura A, Koga R, Arita J et al. Long term outcomes after surgical resection for gastric cancer liver metastasis: an analysis of 64 macroscopically complete resections. Langenbeck Arch Surg 2012; 397: 951-957.

8. Schildberg CW, Croner R, Merkel S, Schellerer V et al. Outcome of operative therapyof hepatic metastasis stomach carcinoma : a retrospective analysis. World J Surg 2012; 36; 872-878.

9. Cheon SH, Rha SY, Jeung HC, Im CK, Kim SH, Kim HR et al. Survival benefit of combined curative resection of the stomach /D2 resection) and liver in gastric cancer patients with liver metastases. Ann Oncol 2008; 19: 1146-1153.

10. Kinoshita T, Kinoshita T, Saiura A, Esaki M, Sakamoto H, Yamanaka T et al. Multicentre analysis of long-term outcome after surgical resection for gastric cancer liver metastases. Br J Surg 2015; 102: 102-107.

11. Takemura N, Saiura A, Koga R, Yoshioka R, Yamamoto J, Kokudo $\mathbf{N}$ et al. Repeat hepatectomy for recurrent liver metastasis from gastric carcinoma. World J Surg 2013; 37: 2664-2670.

12. Yoshida M, Ohtsu A, Boku N, Miyata Y, Shirao K, Shimada Y et al. Long-term survival and prognostic factors in patients with metastatic gastric cancer treated with chemotherapy in the Japan Clinical Oncology Group (JCOG) study. Jpn J Clin Oncol 2004; 34: 654-659.

13. Chen J, Tang Z, Dong X, Gao S, Fang H, Wu D et al. Radiofrequency ablation for liver metastasis from gastric cancer. Eur J Surg Oncol 2013; 39: 701-706. 